\title{
An application of prophet regions to optimal stopping with a random number of observations
}

\author{
Pieter C. Allaart \\ University of North Texas *
}

April 6, 2004

\begin{abstract}
Let $X_{1}, X_{2}, \ldots$ be any sequence of nonnegative integrable random variables, and let $N \in\{1,2, \ldots\}$ be a random variable with known distribution, independent of $X_{1}, X_{2}, \ldots$ The optimal stopping value $\sup _{t} E\left(X_{t} I(N \geq t)\right)$ is considered for two players: one who has advance knowledge of the value of $N$, and another who does not. Sharp ratio and difference inequalities relating the two players' optimal values are given in a number of settings. The key to the proofs is an application of a prophet region for arbitrarily dependent random variables by Hill and Kertz (Trans. Amer. Math. Soc. 278, 197-207 (1983)).
\end{abstract}

AMS 2000 subject classification: 60G40, 62L15.

Key words: Optimal stopping, prophet inequality, random horizon

${ }^{*}$ Author's address: Mathematics Department, University of North Texas, P.O. Box 311430, Denton, TX 76203-1430; e-mail: allaart@unt.edu 


\section{Introduction}

This paper compares the optimal stopping values of two players possessing different degrees of information in a random horizon model. More precisely, let $X_{1}, X_{2}, \ldots$ be any sequence of nonnegative integrable random variables, and let $N$ be a random variable with known distribution, independent of $X_{1}, X_{2}, \ldots$ and taking values in the positive integers. Suppose two players sequentially observe the random variables $X_{1}, \ldots, X_{N}$. Each player can stop at any stage $j$, and will then receive $X_{j}$ if $N \geq j$, or zero if $N<j$. Assume that one player, henceforth to be called the informed gambler, knows the value of $N$ before seeing the first observation, whereas the other player, to be called the uninformed gambler, knows only the distribution of $N$, but not its actual value, and hence runs a real risk of rejecting the last observation and ending up with nothing. How widely can their optimal expected returns diverge?

We will give sharp inequalities relating the two players' optimal values in the following three settings:

(i) $N$ is almost-surely bounded;

(ii) The $X_{j}$ are uniformly bounded;

(iii) $N$ is almost-surely bounded and the $X_{j}$ are uniformly bounded.

The key to deriving these results is to show that the optimal values of the two players can be "sandwiched" between the values of a prophet and a gambler in an associated classical prophet problem. In a classical prophet problem, the expected return of a prophet, who has complete foresight into the future, is compared with that of a gambler, who has no such foresight. Hence if a sequence of random variables $Y_{1}, \ldots, Y_{n}$ is observed, a comparison of prophet and gambler comes down to comparing $M:=\mathrm{E}\left(\max _{j \leq n} Y_{j}\right)$ and $V:=\sup _{t} \mathrm{E} Y_{t}$, where $t$ ranges over all (nonanticipatory) stopping rules for $Y_{1}, \ldots, Y_{n}$. Prophet inequalities have been a popular area of study since Krengel and Sucheston [11] published their celebrated inequality 
( $M<2 V$ if $Y_{1}, \ldots, Y_{n}$ are independent and nonnegative). Dozens of extensions and variations have been published since, many of which are mentioned in the survey paper by Hill and Kertz [9]. A more recent overview is the book by Harten et al. [6], along with its revised and translated version by Schmitz [15]. However, new results have continued to appear at a steady rate to this date.

To solve the problem of the informed and the uninformed gambler, it will be shown that the informed gambler's advantage (both proportionate and difference-wise) is bounded above by the advantage of a prophet over a gambler in optimal stopping of the sequence $Y_{j}=X_{j} I(N \geq j)$. Standard prophet inequalities for arbitrarily dependent random variables by Hill and Kertz [8] then immediately carry over to the informed/uninformed gambler case. Finally, concrete examples will be given to show that the bounds are sharp. In case (iii), for instance, the extremal case is when the $X_{j}$ are degenerate random variables that form an increasing geometric sequence, and the distribution of $N$ is truncated geometric in such a way that the process $\left\{Y_{j}\right\}$ is a martingale. Similar distributions are $\varepsilon$-extremal in cases (i) and (ii), where the best-possible inequality is strict. Since the random variables $X_{1}, X_{2}, \ldots$ in the extremal cases are independent, the same inequalities are sharp for the smaller class of independent random variables.

The optimal stopping problem with a random number of observations has been considered by a number of authors, but mostly in the framework of the best choice or secretary problem, where the objective is to maximize the probability of choosing the best among an unknown number $N$ of candidates. For example, Presman and Sonin [12] and Rasmussen and Robbins [13] assume, as in the present note, that $N$ is a random variable with known distribution. On the other hand, Hill and Krengel [10] assume that $N$ is chosen by an opponent so as to minimize the player's probability of success, giving minimax strategies for the player and the opponent. A different approach, where candidates arrive at i.i.d. random times, was studied by Bruss [3] and Bruss and Samuels [4]. Note that in all of these papers, the objective is different 
from the objective considered here, which is to maximize the expected value of the selected observation.

More generally, the random horizon model can be viewed as a special case of optimal stopping with partial information. The most commonly studied type of partial information is that of unknown distributions, and Allaart and Monticino [1] and Saint-Mont [14] have proved prophet-like inequalities comparing the return of a player who knows the distributions of the random variables to that of a player who does not. Exploring yet another type of partial information, Assaf et al. extend well-known prophet inequalities to a model where only a noise-corrupted version of the random variables can be observed.

\section{Notation and Results}

In order to give formal statements of the main results, some notation is needed. For a sequence of random variables $Y_{1}, Y_{2}, \ldots$, let $V\left(Y_{1}, Y_{2}, \ldots\right)=\sup _{t} \operatorname{E} Y_{t}$ and $V\left(Y_{1}, \ldots, Y_{j}\right)=\sup _{t}\left\{\mathrm{E} Y_{t}: t \leq j\right\}$, where $t$ ranges over the set of all stopping rules measurable with respect to the natural filtration of $Y_{1}, Y_{2}, \ldots$ For $j=1,2 \ldots$, introduce the sigma algebras

$$
\mathcal{F}_{j}=\sigma\left\{X_{1} I(N \geq 1), \ldots, X_{j} I(N \geq j)\right\}
$$

and

$$
\mathcal{F}_{j}^{+}=\sigma\left\{X_{1}, \ldots, X_{j}, N\right\}
$$

(where $I(A)$ denotes the indicator random variable of the event $A$ ). Note that our choice of $\mathcal{F}_{j}$ reflects the view that the uninformed gambler will not know when the final observation has been reached: after the $N$ th observation, he will merely see a string of zeros, not knowing whether these are due to zero values among the $X_{j}$, or to the time horizon having been reached. Instead, we could use the larger sigma algebra $\widehat{\mathcal{F}}_{j}=\sigma\left\{X_{1}, I(N \geq 1), \ldots, X_{j}, I(N \geq j)\right\}$. With this choice, the uninformed gambler 
would possess more information, but his optimal stopping value would nonetheless be the same. Thus, the choice is immaterial.

Let $\mathcal{T}$ be the set of stopping rules adapted to the filtration $\left\{\mathcal{F}_{j}\right\}_{j \geq 1}$, and let $\mathcal{T}^{+}$ be the set of stopping rules adapted to the filtration $\left\{\mathcal{F}_{j}^{+}\right\}_{j \geq 1}$. Note that $\mathcal{T}$ contains those stopping rules available to the uninformed gambler, whereas $\mathcal{T}^{+}$consists of those stopping rules available to the informed gambler. Define

$$
V_{U}:=\sup \left\{\mathrm{E}\left(X_{t} I(N \geq t)\right): t \in \mathcal{T}\right\}
$$

and

$$
V_{I}:=\sup \left\{\mathrm{E}\left(X_{t} I(N \geq t)\right): t \in \mathcal{T}^{+}\right\}
$$

Since $\mathcal{F}_{j} \subseteq \mathcal{F}_{j}^{+}$, it is obvious that $V_{U} \leq V_{I}$. Converse inequalities are given by the following theorems.

Theorem 2.1 If $N \leq n$ a.s. for some integer $n \geq 2$, and if $\mathrm{P}\left(\max \left\{X_{1}, \ldots, X_{N}\right\}>\right.$ 0) $>0$, then

$$
V_{I}<n V_{U}
$$

and this bound is sharp.

Theorem 2.2 If $X_{j} \in[a, b]$ for all $j$ (where $0 \leq a<b$ ), then

$$
V_{I}-V_{U}< \begin{cases}b e^{-1}, & \text { if } a / b \leq e^{-1} \\ a \ln (b / a), & \text { otherwise. }\end{cases}
$$

If, furthermore, $N \leq n$ a.s., then

$$
V_{I}-V_{U} \leq \begin{cases}b(1-1 / n)^{n}, & \text { if } a / b \leq(1-1 / n)^{n-1} \\ a(n-1)\left(1-(a / b)^{1 /(n-1)}\right), & \text { otherwise. }\end{cases}
$$

All bounds are sharp, and the bounds in (3) are attained. 
The bounds given in inequalities (2) and (3) can be interpreted as the maximum amounts one should be willing to pay for the privilege of being told in advance the number of available observations. For example, suppose there is no upper bound on $N$, but the random variables $X_{1}, X_{2}, \ldots$ are known to take values in $\left[\frac{1}{2}, 1\right]$. Then inequality $(2)$ implies that one should never pay more than $(\ln 2) / 2 \approx .3466$ for information about the total number of observations.

Example 2.3 A job seeker, fresh from college, has submitted 25 job applications. An unknown number $N$ of these will result in job offers. Assume for the sake of simplicity that the job seeker aims to maximize his expected starting salary, and that for a new graduate with the qualifications of the job seeker, starting salaries range from $\$ 50,000$ to $\$ 70,000$. From statistical job market data, the job seeker knows both the distribution of $N$ and the distribution of the prospective starting salaries. If every offer must be accepted or declined immediately as it comes in, how much more could the job seeker have expected to make if he could foretell the exact number of job offers to be extended to him?

Using inequality (3) with $n=25, a=50,000$ and $b=70,000$ we find that in the worst of all cases, this difference is $50,000 \cdot 24 \cdot\left(1-(5 / 7)^{1 / 24}\right) \approx \$ 16706$.

\section{Proofs}

For $j=1,2, \ldots$, define

$$
Y_{j}=X_{j} I(N \geq j) .
$$

The first lemma makes precise the claim from the introduction that the optimal values $V_{U}$ and $V_{I}$ are "sandwiched" between the values $V$ and $M$ of the classical prophet problem associated with the sequence $\left\{Y_{j}\right\}$.

Lemma $3.1 \quad$ (i) $V_{U}=V\left(Y_{1}, Y_{2}, \ldots\right)$, and $V_{I} \leq \mathrm{E}\left(\sup _{j \geq 1} Y_{j}\right)$.

(ii) If $N \leq n$ a.s., then $V_{U}=V\left(Y_{1}, \ldots, Y_{n}\right)$, and $V_{I} \leq \mathrm{E}\left(\max \left\{Y_{1}, \ldots, Y_{n}\right\}\right)$. 
Proof. That $V_{U}=V\left(Y_{1}, Y_{2}, \ldots\right)$ follows immediately from the definition of $V_{U}$. The inequality for $V_{I}$ can be seen by observing that the informed gambler on the set $\{N=j\}$ can employ the stopping rule optimal for the sequence $X_{1}, \ldots, X_{j}$. Using the independence of $N$ from $X_{1}, X_{2}, \ldots$, this gives

$$
\begin{aligned}
V_{I} & =\sum_{j=1}^{\infty} V\left(X_{1}, \ldots, X_{j}\right) \mathrm{P}(N=j) \\
& \leq \sum_{j=1}^{\infty} \mathrm{E}\left(\max \left\{X_{1}, \ldots, X_{j}\right\}\right) \mathrm{P}(N=j) \\
& =\mathrm{E}\left(\max \left\{X_{1}, \ldots, X_{N}\right\}\right) \\
& =\mathrm{E}\left(\sup _{j \geq 1} Y_{j}\right) .
\end{aligned}
$$

Statement (ii) follows from (i) since, if $N \leq n$ a.s., then $Y_{j}=0$ for all $j>n$.

The next lemma recollects the prophet inequalities on which the results of this paper are based. Variations of the first inequality have appeared in [7] and [8]. However, it does not seem to have been stated explicitly in the stronger form below.

Lemma 3.2 (i) Let $n \geq 2$, and let $Y_{1}, \ldots, Y_{n}$ be any sequence of nonnegative, integrable random variables such that $\mathrm{P}\left(\max _{j \leq n} Y_{j}>0\right)>0$. Then

$$
\mathrm{E}\left(\max _{j \leq n} Y_{j}\right)<n V\left(Y_{1}, \ldots, Y_{n}\right) .
$$

(ii) [Hill and Kertz [8], Theorem 3.2.] Let $Y_{1}, \ldots, Y_{n}$ be any sequence of random variables taking values in $[0,1]$, and let $V\left(Y_{1}, \ldots, Y_{n}\right)=x$. Then

$$
\mathrm{E}\left(\max _{j \leq n} Y_{j}\right) \leq x\left(1+(n-1)\left(1-x^{1 /(n-1)}\right)\right),
$$

and this bound is attained.

(iii) [Hill and Kertz [8], Theorem 4.2.] Let $Y_{1}, Y_{2}, \ldots$ be any sequence of random variables taking values in $[0,1]$, and let $V\left(Y_{1}, Y_{2}, \ldots\right)=x$. Then

$$
\mathrm{E}\left(\sup _{j \geq 1} Y_{j}\right) \leq x-x \ln x
$$

This bound is sharp, and holds with strict inequality if $0<x<1$. 
Proof of (i). Observe first that $\mathrm{E}\left(\max Y_{j}\right) \leq \sum \mathrm{E} Y_{j} \leq n \max \mathrm{E} Y_{j} \leq n V\left(Y_{1}, \ldots, Y_{n}\right)$. Suppose the two sides of (4) are equal. Then $\mathrm{E}\left(\max Y_{j}\right)=\mathrm{E}\left(\sum Y_{j}\right)$, and therefore $\max Y_{j}=\sum Y_{j}$ a.s. But this means that with probability one, there is an index $j_{0}$ such that $Y_{j}=0$ for all $j \neq j_{0}$. It follows that the rule $t=\min \left\{j: Y_{j}>0\right\}$ (or $=n$ if $\left.\max _{j \leq n} Y_{j}=0\right)$ is optimal, and thus $V\left(Y_{1}, \ldots, Y_{n}\right)=\mathrm{E} Y_{t}=\mathrm{E}\left(\max Y_{j}\right)=$ $n V\left(Y_{1}, \ldots, Y_{n}\right)$, a contradiction since $n \geq 2$ and the hypothesis of the proposition implies $\mathrm{E}\left(\max Y_{j}\right)>0$. Hence the inequality is strict.

Note that the weak-inequality version of (4) appears in Proposition 1 of Hill and Kertz [7]. The result with strict inequality is given in Corollary 3.5 of Hill and Kertz [8], but under the unnecessary hypothesis that $Y_{j} \in[0,1]$.

Proof of Theorem 2.1. The inequality (1) follows directly from Lemma 3.1 and Lemma 3.2 (i). To see that the bound is sharp, let $K$ be a constant greater than 1, and let $X_{j}=K^{j}$ for all $j$. Let $\mathrm{P}(N=j)=\left(K^{-1}\right)^{j-1}\left(1-K^{-1}\right)$ for $1 \leq j \leq n-1$, and $\mathrm{P}(N=n)=\left(K^{-1}\right)^{n-1}$. Then $V\left(X_{1}, \ldots, X_{j}\right)=\mathrm{E} X_{j}=K^{j}$ for all $j$, so that

$$
\begin{aligned}
V_{I} & =\sum_{j=1}^{n} \mathrm{P}(N=j) V\left(X_{1}, \ldots, X_{j}\right) \\
& =\sum_{j=1}^{n-1}\left(K^{-1}\right)^{j-1}\left(1-K^{-1}\right) K^{j}+\left(K^{-1}\right)^{n-1} K^{n} \\
& =n K-(n-1) .
\end{aligned}
$$

On the other hand, one checks easily that the process $Y_{j}=X_{j} I(N \geq j)$ forms a martingale, and thus $V_{U}=V\left(Y_{1}, \ldots, Y_{n}\right)=\mathrm{E} Y_{1}=K$. Therefore,

$$
\frac{V_{I}}{V_{U}}=n-\frac{n-1}{K},
$$

and letting $K \rightarrow \infty$ shows that (1) is sharp.

Proof of Theorem 2.2. Note that it is sufficient to prove the theorem for the case $b=1$, since the general case then follows by a simple rescaling. Only the proof of (3) will be given here. The proof of (2), which is analogous, is left to the reader. 
Assume then that $X_{j} \in[a, 1]$, and that $N \leq n$ almost surely. Lemma 3.2 (ii) implies that if $V_{U}=V\left(Y_{1}, \ldots, Y_{n}\right)=x$, then

$$
V_{I} \leq \operatorname{E} \max \left\{Y_{1}, \ldots, Y_{n}\right\} \leq x\left(1+(n-1)\left(1-x^{1 /(n-1)}\right)\right)
$$

and hence

$$
V_{I}-V_{U} \leq x(n-1)\left(1-x^{1 /(n-1)}\right)
$$

Note that the right hand side of (6) is a unimodal function of $x$, with a unique maximum at $x^{*}=(1-1 / n)^{n-1}$. Since the hypothesis $X_{j} \in[a, 1]$ implies that $x \geq a$, $V_{I}-V_{U}$ is maximized at $x=x^{*}$ if $a \leq x^{*}$, and at $x=a$ otherwise. Substituting these values back into (6) gives (3) for the case $b=1$.

To show that the bound is attained, define

$$
q=\left\{\begin{array}{lll}
1-1 / n, & \text { if } & a \leq x^{*} \\
a^{1 /(n-1)}, & \text { if } & a>x^{*}
\end{array}\right.
$$

Let $X_{j} \equiv q^{n-j}$ for $j=1,2 \ldots, n$, let $\mathrm{P}(N=j)=q^{j-1}(1-q)$ for $1 \leq j \leq n-1$, and let $\mathrm{P}(N=n)=q^{n-1}$. Then the sequence $\left\{Y_{j}\right\}$ defined by $Y_{j}=X_{j} I(N \geq j)$ is a martingale, and a calculation similar to that in the proof of Theorem 2.1 shows that

$$
V_{I}-V_{U}= \begin{cases}(1-1 / n)^{n}, & \text { if } \quad a \leq x^{*} \\ a(n-1)\left(1-a^{1 /(n-1)}\right), & \text { if } \quad a>x^{*} .\end{cases}
$$

Finally, the sharpness of (2) follows from that of (3) by letting $n \rightarrow \infty$.

Remark 3.3 The martingale $\left\{Y_{j}\right\}$ occurring in the above proof was given by Dubins and Pitman [5] as the extremal process attaining their maximal inequality for martingales. The same process was used by Hill and Kertz [8] to demonstrate the sharpness of inequality (5).

Remark 3.4 Note that the extremal sequences $X_{1}, X_{2}, \ldots$ given in the above proofs are in fact independent. Thus, the inequalities in Theorems 2.1 and 2.2 would remain 
best possible if the strictly smaller class of sequences of independent random variables were being considered. This makes the type of comparison considered here quite different from classical prophet inequalities, where independence usually reduces the prophet's advantage significantly.

Remark 3.5 Since the proofs of Theorems 2.1 and 2.2 are based on Lemmas 3.1 and 3.2 , it is easy to check that the inequalities (1)-(3) remain valid if $V_{I}$ is replaced with the (potentially larger) $\mathrm{E}\left(\sup _{j \geq 1} Y_{j}\right)$. Thus, an 'absolute prophet' who has advance knowledge of both $N$ and $X_{1}, X_{2}, \ldots$ would, in the extremal case, not have a greater advantage over the uninformed gambler than the informed gambler has. In this sense, advance knowledge of the number of available observations is actually more empowering than advance knowledge of the observations themselves.

\section{Acknowledgement}

The author thanks two anonymous referees for several helpful suggestions.

\section{References}

[1] Allaart, P. and Monticino, M. (2003). Pseudo-prophet inequalities in averageoptimal stopping, Sequential Anal. 22, 233-239.

[2] Assaf, D., Goldstein, L. and Samuel-Cahn, E. (1998). A statistical version of prophet inequalities. Ann. Stat. 26, 1190-1197.

[3] Bruss, F. (1984). A unified approach to a class of best choice problems with an unknown number of options, Ann. Probab. 12, 882-889.

[4] Bruss, F. and Samuels, S. (1987). A unified approach to a class of optimal selecting problems with an unknown number of options, Ann. Probab. 15, 824830.

[5] Dubins, L. E. and Pitman, J. (1980). A maximal inequality for skew fields. $Z$. Wahrsch. Verw. Gebiete 52, 219-227. 
[6] Harten, F., Meyerthole, A. and Schmitz, N. (1997). Prophetentheorie. Teubner Skripten zur Mathematischen Stochastik. Teubner-Verlag, Stuttgart. (in German)

[7] Hill, T. P. and Kertz, R. P. (1981). Ratio comparisons of supremum and stop rule expectations. Z. Wahrsch. Verw. Gebiete 56, 283-285.

[8] Hill, T. P. and Kertz, R. P. (1983). Stop rule inequalities for uniformly bounded sequences of random variables. Trans. Amer. Math. Soc. 278, 197-207.

[9] Hill, T. P. and Kertz, R. P. (1992). A survey of prophet inequalities in optimal stopping theory. Contemp. Math. 125, 191-207.

[10] Hill, T. and Krengel, U. (1991). Minimax-optimal stop rules and distributions in secretary problems, Ann. Probab. 19, 342-353.

[11] Krengel, U. and Sucheston, L. (1978). On semiamarts, amarts, and processes with finite value. Probability on Banach Spaces, Ed. by J. Kuelbs, Marcel Dekker, New York.

[12] Presman, E. and Sonin, I. (1972). The best choice problem for a random number of objects, Theory Probab. Applic. 17, 657-668.

[13] Rasmussen, W. and Robbins, H. (1975). The candidate problem with unknown population size, J. Appl. Probab. 12, 692-701.

[14] Saint-Mont, U. (2003). Comparison of Values of Independent Random Variables in a Bayesian Setting, Sequential Anal. 22, 159-165.

[15] Schmitz, N. (2000). Prophet theory. Skripten zur Mathematischen Statistik, Nr. 34. Teubner-Verlag, Stuttgart. 